\section{Ultrahigh-yield on-surface synthesis and assembly of circumcoronene into a chiral electronic Kagome-honeycomb lattice}

\author{
Mykola Telychko ${ }^{1 *}$, Guangwu Li $^{1 *}$, Pingo Mutombo ${ }^{2,3}$, Diego Soler-Polo ${ }^{4}$, Xinnan Peng ${ }^{1}$, Jie Su$^{1}$, \\ Shaotang Song ${ }^{1}$, Ming Joo Koh ${ }^{1}$, Mark Edmonds ${ }^{5}$, Pavel Jelínek ${ }^{2,6 \dagger}$, Jishan Wu ${ }^{1 \dagger}$, Jiong Lu ${ }^{1,7 \dagger}$
}

On-surface synthesis has revealed remarkable potential in the fabrication of atomically precise nanographenes. However, surface-assisted synthesis often involves multiple-step cascade reactions with competing pathways, leading to a limited yield of target nanographene products. Here, we devise a strategy for the ultrahigh-yield synthesis of circumcoronene molecules on $\mathrm{Cu}(111)$ via surface-assisted intramolecular dehydrogenation of the rationally designed precursor, followed by methyl radical-radical coupling and aromatization. An elegant electrostatic interaction between circumcoronenes and metallic surface drives their self-organization into an extended superlattice, as revealed by bond-resolved scanning probe microscopy measurements. Density functional theory and tight-binding calculations reveal that unique hexagonal zigzag topology of circumcoronenes, along with their periodic electrostatic landscape, confines two-dimensional electron gas in $\mathrm{Cu}(111)$ into a chiral electronic Kagome-honeycomb lattice with two emergent electronic flat bands. Our findings open up a new route for the high-yield fabrication of elusive nanographenes with zigzag topologies and their superlattices with possible nontrivial electronic properties.

\section{INTRODUCTION}

On-surface synthesis has emerged as a powerful strategy for the bottom-up fabrication of low-dimensional nanographenes (NGs) with atomic precision (1-5). The core concept of the surface-assisted synthetic strategy relies on the rational design of the elegant molecular precursors that subsequently undergo chemical transformation along a certain reaction pathway toward the desired NG product. The versatility of this synthetic strategy has been exemplified by the seminal fabrication of a wealth of open-shell and closed-shell NGs. In addition, the electronic, magnetic, and optical properties of NGs can be precisely tuned via engineering their chemical structure and/ or concomitant $\pi$-electron topology of the NG (6-14). Therefore, on-surface fabricated NGs with tailored physicochemical properties represent highly prosperous functional materials for next-generation quantum device applications (15-18).

However, on-surface synthetic strategies have long been constrained by a low scalability due to a rather limited yield of target NGs. Conventional surface-assisted synthetic routes often involve a series of cascade reactions with competing reaction pathways, which inevitably lead to the formation of numerous undesired products. The limited yield of target products imposes a formidable challenge toward the practical applications of the NGs synthesized on the surface, because feasibility of the large-scale (i.e., high-yield) fabrication is

\footnotetext{
'Department of Chemistry, National University of Singapore, 3 Science Drive 3, Singapore 117543, Singapore. ${ }^{2}$ Institute of Physics, The Czech Academy of Sciences, 16200 Prague, Czech Republic. ${ }^{3}$ Department of Petrochemistry and Refining, University of Kinshasa, Kinshasa, Democratic Republic of Congo. ${ }^{4}$ Universidad Autónoma de Madrid, Campus Cantoblanco, Madrid, Spain. ${ }^{5}$ School of Physics and Astronomy, Monash University, Clayton, Victoria, Australia. ${ }^{6}$ Regional Centre of Advanced Technologies and Materials, Palacký University, 78371 Olomouc, Czech Republic. ${ }^{7}$ Centre for Advanced 2D Materials (CA2DM), National University of Singapore, 6 Science Drive 2, Singapore 117546, Singapore.

*These authors contributed equally to this work.

†Corresponding author. Email: chmluj@nus.edu.sg (J.L.); jelinekp@fzu.cz (P.J.);chmwuj@ nus.edu.sg (J.W.)
}

vital for their technological relevance. To overcome this issue, it is required to devise a new synthetic route with a new conceptual design of precursor molecules, which allows for their robust transformation toward target NGs via suppressing the side reactions, including undesired intermolecular coupling and intramolecular transformations.

To this end, we design a novel surface-assisted synthetic route for the ultrahigh-yield fabrication of a hexagonal zigzag-edged NG, namely, circumcoronene, on the $\mathrm{Cu}(111)$ surface. Our surface-assisted cyclodehydrogenation strategy relies on the robust dehydrogenative coupling of the methyl groups at the adjacent sites of the rationally designed precursor molecule II, followed by dehydrogenative aromatization on metallic substrate (Fig. 1A). Because of a remarkably high-yield synthesis and elegant molecule-substrate interaction, circumcoronene molecules self-assemble into extended superlattices (Fig. 1D). The structural and electronic properties of both individual molecules and their superlattices are characterized via bond-resolved noncontact atomic force microscopy (ncAFM) and scanning tunneling microscopy (STM)/scanning tunneling spectroscopy (STS), respectively. We demonstrate that the circumcoronene superlattice confines the two-dimensional (2D) electron gas on $\mathrm{Cu}(111)$ into an extended Kagome-honeycomb electronic lattice (Fig. 1D) with a two-flat-band electronic structure, in contrast to a conventional electronic Kagome lattice with a single dispersionless band. Our findings open a new route for both ultrahigh-yield synthesis of elusive NGs and precise fabrication of artificial 2D lattices with possible nontrivial electronic properties toward future technological applications.

\section{RESULTS AND DISCUSSION}

\section{Surface-assisted synthesis of circumcoronene}

To fabricate a circumcoronene, we adopt a strategy of a surfaceassisted cyclodehydrogenation of the rationally designed precursor molecule II $\left(\mathrm{C}_{54} \mathrm{H}_{42}\right)$ that was obtained via multistep wet-chemistry synthesis (Fig. 1A). Oxidative cyclodehydrogenation 

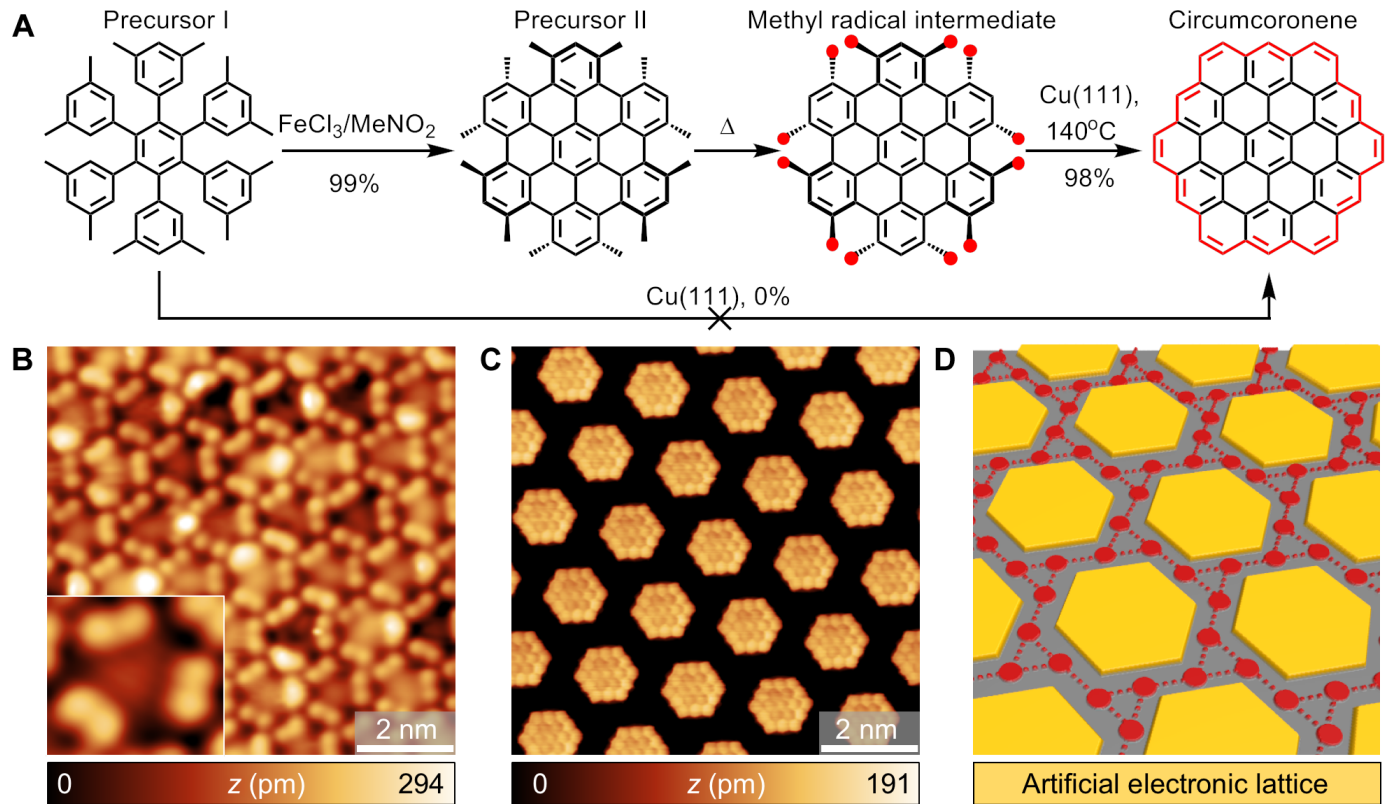

Fig. 1. On-surface synthesis of circumcoronene-based superlattice. (A) Precursor molecule II undergoes cyclodehydrogenation on Cu(111) via methyl radical-radical coupling toward formation of the circumcoronene. The cyclodehydrogenation results in ultrahigh-yield circumcoronene molecules. (B) STM image acquired after deposition of the precursor II onto $\mathrm{Cu}(111)$ at room temperature. Inset shows the STM image of an individual precursor molecule. (C) STM image collected after sample annealing at $140^{\circ} \mathrm{C}$, revealing the presence of the circumcoronene-based self-assembly on $\mathrm{Cu}(111)$. (D) Schematic illustration of the chiral Kagome-honeycomb lattice.

of the hexakis(3,5-dimethylphenyl)benzene precursor molecule I by iron(III) chloride gave the hexa-peri-hexabenzocoronene (HBC) precursor molecule II carrying 12 methyl groups at the bay regions in nearly quantitative yield. This additional step reduces the rotational degree of freedom of six pairs of methyl groups and locks their positions ready for the subsequent dehydrogenative coupling into circumcoronene with zigzag-edged topology on the catalytically active $\mathrm{Cu}(111)$ surface. Such a precursor design prevents the undesired intramolecular and intermolecular side reactions, allowing for high-yield synthesis of zigzag-edged NGs. Furthermore, the presence of methyl groups in precursor II reduces intermolecular $\pi-\pi$ interactions within the compound and thus facilitates in situ sublimation for on-surface synthesis. A complete synthetic route of II along with standard structural characterization is presented in the Supplementary Materials (note S1). The conformation and self-assembly of this molecule on $\mathrm{Ag}(111)$ and $\mathrm{Au}\left(\begin{array}{lll}1 & 12 & 12\end{array}\right)$ were previously investigated $(19,20)$, but its chemical reaction on metal surface was not reported.

Precursor II was then deposited onto the $\mathrm{Cu}(111)$ surface held at room temperature via thermal sublimation from a Knudsen cell under ultrahigh vacuum (UHV) conditions. Upon deposition onto the $\mathrm{Cu}(111)$ surface, precursor molecules self-assemble into close-packed ordered structures (Fig. 1B). Precursor II adopts a nonplanar saddle-shape conformation geometry $(20,21)$, which results in the triangular-shaped topography in STM images (inset in Fig. 1B). Subsequently, the transformation of precursor II into the target circumcoronene was achieved by thermal annealing at $\sim 140^{\circ} \mathrm{C}$ for 1 hour, whereby the intramolecular cyclodehydrogenation of the methyl groups can be triggered. The chemical transformation of precursor II into the final product involves two major steps, namely, the dehydrogenative coupling of the adjacent methyl groups followed by dehydrogenative aromatization on $\mathrm{Cu}(111)$ (fig. S5), leading to a remarkably high product yield (>98\%) (Fig. 1C), in stark contrast to the case of precursor I (unsuccessful, fig. S7). Note that the product yield is determined by a statistical analysis of multiple large-area STM images $\left(2000 \mathrm{~nm}^{2}\right)$ (refer to fig. S6 for details).

\section{Characterization of single circumcoronenes and their superlattice}

A high-yield synthesis allows individual circumcoronene molecules to self-organize into extended superlattices characterized by a $\sqrt{3} \times \sqrt{3}$ elementary unit cell with a lattice constant of $18.4 \AA$ (Fig. 2A). It is noted that the average-sized superlattice domain contains $\sim 330$ molecules, which occupy a total area of $\sim 1000 \mathrm{~nm}^{2}$ (the largest domains observed consist of $\sim 520$ molecules). To determine an accurate intramolecular structure of individual molecules and their superlattices, we conducted a constant-height ncAFM measurement with a carbon monoxide (CO) terminated tip (22-26). The frequency shift $(\Delta f)$ contrast revealed by constant-height ncAFM images reflects the magnitude of short-range forces acting between CO-terminated tip and the sample in a Pauli repulsion regime. Therefore, bright areas with a higher $\Delta f$ value represent regions with an enhanced electron density. A set of ncAFM images collected at various tip-sample heights over individual circumcoronenes (Fig. 2, B and C) unambiguously reveal the molecular skeleton consisting of 19 fused benzene rings with six zigzag edges. Close-up image reveals that the $\mathrm{C}-\mathrm{C}$ bonds at the molecule's corners exhibit the shortest length when imaged at small tip-sample separations (Fig. 2C). At a moderate tip-sample distance (Fig. 2B), the corner bonds also show a brighter ncAFM contrast, suggesting a higher bond order of these $\mathrm{C}-\mathrm{C}$ bonds as compared to the other ones. The calculation of the free-standing circumcoronene based on the B3LYP DFT (density functional theory) functional unambiguously reveals a localization of the shortest $\mathrm{C}-\mathrm{C}$ bonds (or bonds with highest order) at the molecular corners (Fig. 2H). Such 

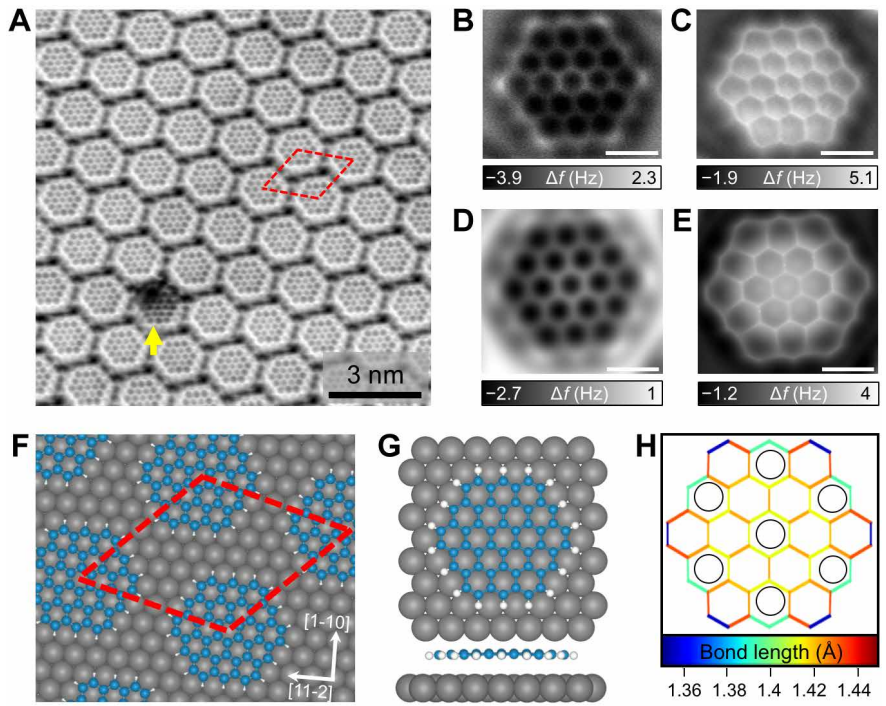

Fig. 2. Bond-resolved ncAFM imaging of the circumcoronene superlattice. (A) Large-scale ncAFM image of the circumcoronene array, collected with a CO-decorated tip. Defective circumcoronene molecule (indicated by yellow arrow) constitutes an approximately $2 \%$ of total products. (B and C) Constant-height ncAFM images of individual circumcoronene molecule, collected at tip-sample distances $z=40 \mathrm{pm}$ in $(\mathrm{B})$ and $z=60 \mathrm{pm}$ in (C) with respect to set point $V=20 \mathrm{mV} ; l=1$ nA. ( $D$ and $\mathbf{E}$ ) ncAFM images simulated using a numerical probe-particle approach, for a physical tip-sample distance of $0 \mathrm{pm}$ in (E) and $20 \mathrm{pm}$ in (D) with respect to set point $14.4 \AA$. . (F) DFT-relaxed model of the circumcoronene-based superlattice on $\mathrm{Cu}(111)$. (G) Top view and side view of the single circumcoronene molecule on $\mathrm{Cu}(111)$. (H) DFT (B3LYP) calculations of the bond lengths within the circumcoronene molecule. Scale bars (B to E), 500 pm.

a peculiar bond localization within circumcoronene can be described on the basis of Clar's aromatic sextet rule that predicts the formation of the maximal number of aromatic sextets (seven in circumcoronene) with a $\mathrm{C}=\mathrm{C}$ localization at the molecular corners. ncAFM contrast of peripheral $\mathrm{C}-\mathrm{C}$ bonds in circumcoronene associated with an increased bond localization of aromatic rings also resembles that of a Kekulene molecule (27).

To uncover the atomic registry of the circumcoronene-based superlattice on $\mathrm{Cu}(111)$, we imaged both individual molecules within the superlattice and adjacent $\mathrm{Cu}(111)$ lattice, respectively (fig. S8). Superimposing the $\mathrm{Cu}(111)$ lattice over the circumcoronene array reveals the adsorption sites of all the circumcoronene molecules corroborated by the DFT-predicted model (Fig. 2F). Furthermore, DFT calculations also unveil that circumcoronene adopts a slightly bent geometry, with peripheral benzene rings pulled toward the underlying $\mathrm{Cu}(111)$ surface (Fig. 2G). Such a bent structural conformation of circumcoronene is manifested by a brighter contrast of the central benzene ring in the constant-height ncAFM images. The simulated ncAFM images of DFT-relaxed structures using a modified probe-particle model $(28,29)$ reproduce all the key features of experimental ncAFM images including a tip-sample-dependent $\Delta f$ contrast over the corner $\mathrm{C}-\mathrm{C}$ bonds with the highest bond order and higher $\Delta$ fcontrast of the central benzene ring attributed to the nonplanar geometry of the circumcoronene (Fig. 2, D and E).

\section{Electronic properties of the circumcoronene-based superlattice}

We then probed the electronic properties of individual circumcoronene molecules and their superlattice via performing differential conductance spectroscopy $(\mathrm{d} I / \mathrm{d} V)$ measurements (Fig. 3 and fig. S9). In contrast to the rather featureless $\mathrm{d} I / \mathrm{d} V$ spectrum acquired over the bare $\mathrm{Cu}(111)$ surface (only Shockley surface state induced peak around $-0.4 \mathrm{~V}$ ), the $\mathrm{d} I / \mathrm{d} V$ curve collected over circumcoronene at the periphery of the superlattice (blue curve in Fig. 3A) exhibits three characteristic peaks, located at energies of $-1.6 \mathrm{~V},+0.9 \mathrm{~V}$, and $+1.8 \mathrm{~V}$, respectively, resembling that acquired over an isolated molecule (green curve in Fig. $3 \mathrm{~A}$ ). The $\mathrm{d} I / \mathrm{d} V$ curve acquired over the circumcoronene in the interior of the superlattice (red curve in Fig. 3A) reveals additional prominent peaks centered at $+0.36 \mathrm{~V}$ (labeled as $F B_{1}$ ) and $+1.15 \mathrm{~V}$ (labeled as $F B_{2}$ ). We further probed the spatial distribution of these electronic states via $\mathrm{d} I / \mathrm{d} V$ mapping with a CO-functionalized tip. The $\mathrm{d} I / \mathrm{d} V$ maps collected at the corresponding energies of $-1.6 \mathrm{~V},+0.9 \mathrm{~V}$, and $+1.8 \mathrm{~V}$ reveal the characteristic nodal patterns localized over the molecular backbone, which can be assigned to the highest occupied molecular orbital (HOMO), lowest unoccupied molecular orbital (LUMO), and second lowest unoccupied molecular orbital (LUMO+1), respectively, as supported by their excellent resemblance with the calculated $\mathrm{d} I / \mathrm{d} V$ maps of these frontier orbitals using a COdecorated tip $(30,31)$ (insets in Fig. 3, C, E, and G). In contrast, $\mathrm{d} I / \mathrm{d} V$ maps collected at energies of the additional electronic states $\left(F B_{1}\right.$ and $\left.F B_{2}\right)$ within the circumcoronene superlattice reveal remarkable intensity in between molecules (Fig. 3, D and F), which cannot be explicitly ascribed to tunneling into any frontier molecular orbitals. Furthermore, $F B_{1}$ and $F B_{2}$ states largely attenuate at the edge of the circumcoronene superlattice as evident from the $2 \mathrm{D}$ contour plot of $\mathrm{d} I / \mathrm{d} V$ spectra acquired across the superlattice (Fig. 3B).

Flat bands in the chiral electronic Kagome-honeycomb lattice We performed DFT calculation combined with tight-binding (TB) model calculations to understand the physical origin of both $F B_{1}$ and $F B_{2}$ states in the circumcoronene superlattice. These new electronic states arise from the formation of the circumcoronene superlattice that confine $2 \mathrm{D}$ electron gas (2DEG) of the $\mathrm{Cu}(111)$ surface into the chiral electronic Kagome-honeycomb lattice. The calculated electrostatic potential landscape over the circumcoronene superlattice shows a notable accumulation of the negative charge in the plane of circumcoronene (Fig. 4B). This negative electrostatic potential is balanced by a positive potential accumulated in the $\mathrm{Cu}(111)$ surface regions between molecules. In addition, a comparison of the calculated DOS (density of states) plots of the freestanding circumcoronene and $\mathrm{Cu}(111)$-supported circumcoronene shows that the latter exhibits a downshift of the energetic positions of the molecular orbitals (figs. S10 and S11), attributed to the electron transfer from the $\mathrm{Cu}(111)$ substrate to the circumcoronene. Each molecule hosts a negative electrostatic potential and thereby acts as a hexagonally shaped repulsive barrier for creating a periodic modulation and confinement of the 2DEG (32-34). Notably, our DFT calculations also reveal a non-negligible out-of-plane buckling of $\mathrm{Cu}$ atoms in the topmost layer of the $\mathrm{Cu}(111)$ regions forming a periodic buckling pattern (Fig. 4C) reminiscent of the chiral Kagomehoneycomb lattice (Fig. 4A). Because of a computational feasibility, the semi-infinite $\mathrm{Cu}(111)$ surface is represented by only three $\mathrm{Cu}$ layers in our DFT calculations, which therefore cannot provide an accurate description of the electronic structures of surface state and the confined states observed experimentally.

The unique electronic structures of the artificial electronic lattice can be well described using the TB model that involves the electron 


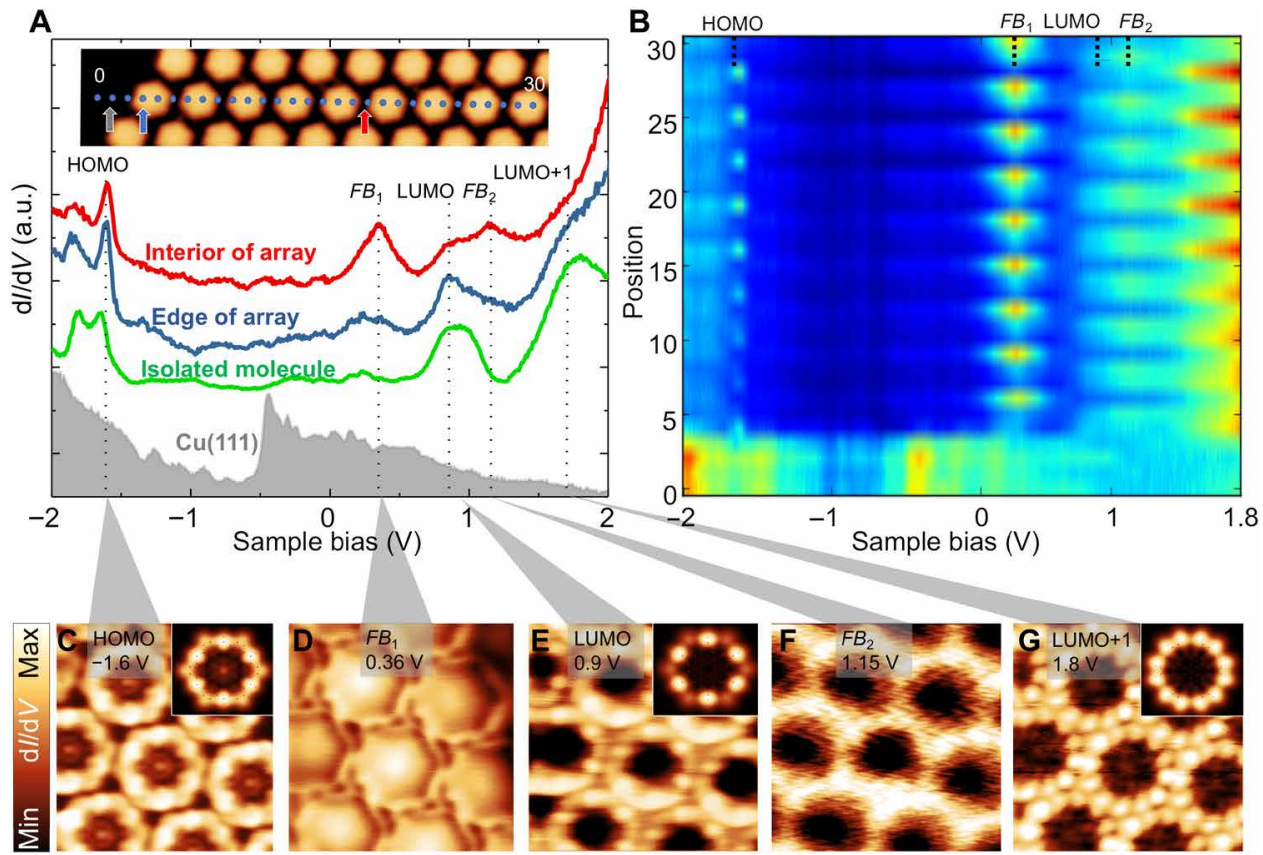

Fig. 3. Electronic structure of the circumcoronene array. (A) $d / / d V$ spectra collected over the bare $\mathrm{Cu}(111)$ substrate (gray), a circumcoronene molecule residing at the periphery of the superlattice (blue), a circumcoronene molecule located in the interior of the superlattice (red), and an isolated molecule (green). a.u., arbitrary units. (B) $2 \mathrm{D}$ contour plot of $\mathrm{d} / \mathrm{d} V$ spectra acquired along the line indicated in the inset in (A). (C to G) Topography STM image ( $1 \mathrm{~V} ; 1 \mathrm{nA})$ and corresponding d//dV maps collected at energetic positions of states HOMO (C), FB $(\mathrm{D})$, LUMO (E), $F B_{2}(\mathrm{~F})$, and LUMO+1 (G). Inset images in (C), (E), and (G) are the calculated d//dV maps of the HOMO (C), LUMO (E), and LUMO+1 (G) of the circumcoronene using a CO-decorated tip.

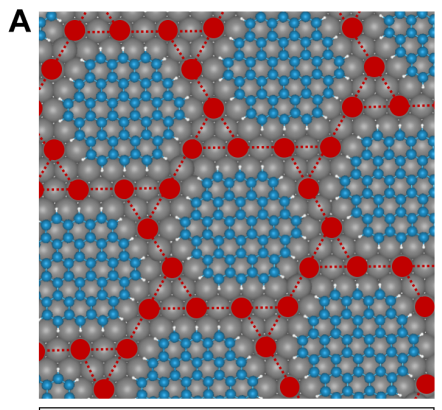

Chiral Kagome-honeycomb lattice

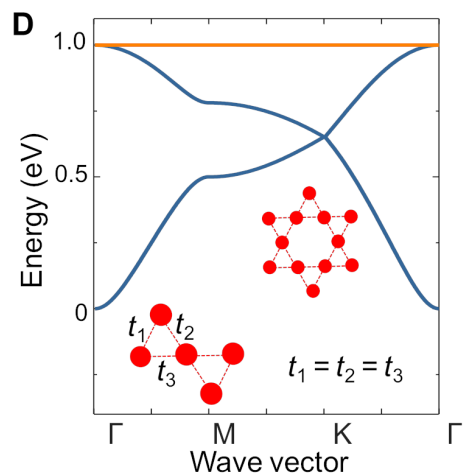

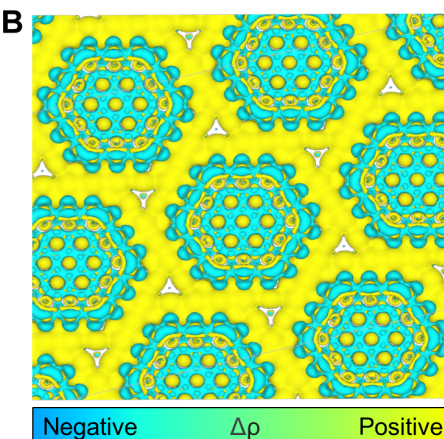

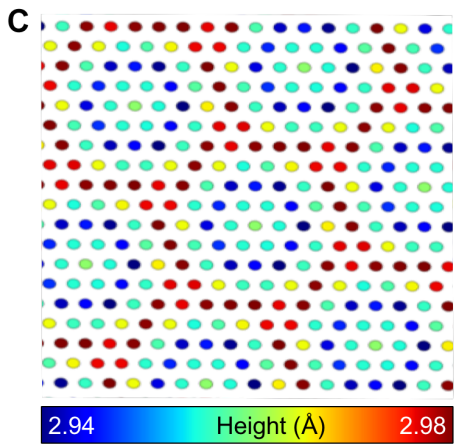

E

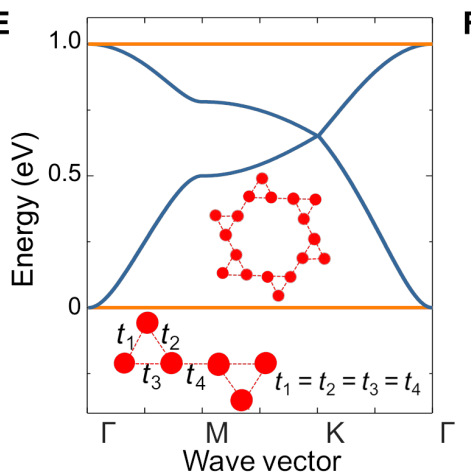

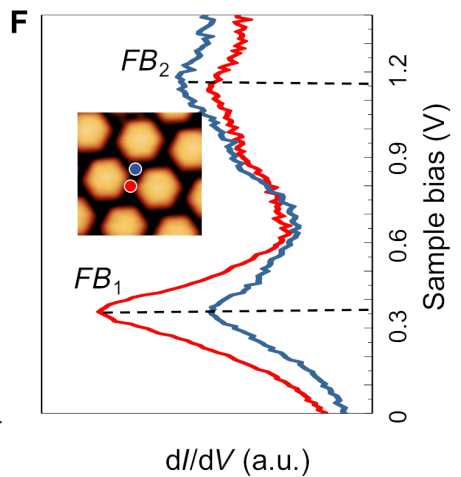

Fig. 4. Origin and electronic structure of the chiral Kagome-honeycomb lattice. (A) Schematic illustration of the chiral Kagome-honeycomb lattice. (B) Electrostatic potential of the circumcoronene superlattice. (C) The out-of-plane buckling of $\mathrm{Cu}$ atoms in the topmost $\mathrm{Cu}(111)$ layer. (D and $\mathbf{E}) \mathrm{A} k$-space dispersion of the electronic bands of the Kagome and chiral Kagome-honeycomb lattices, calculated using the TB model for hopping constants $t_{1}=t_{2}=t_{3}=-0.5 \mathrm{eV}$ (D) and $t_{1}=t_{2}=t_{3}=t_{4}=-0.5 \mathrm{eV}$ (E). (F) Experimental d $/ / \mathrm{d} V$ spectra reveal the existence of two characteristic peaks $\left(F B_{1}\right.$ and $\left.F B_{2}\right)$ associated with two flat bands of the chiral electronic Kagome-honeycomb lattice. 
hopping strength between atomic orbitals associated to lattice sites. TB band structures of a standard Kagome and Kagome-honeycomb lattice are shown in Fig. 4 (D and E, respectively). The common Kagome lattice is composed of a network of corner-sharing triangles, leading to the destructive phase interference of electron hopping pathways between nearest-neighbor lattice sites. As such, it precludes electron hopping between neighboring unit cells and results in the localization of electronic state within a single Kagome hexagon $(35,36)$. Such a unique real-space localization of the electronic state further manifests itself by the formation of the empty state dispersionless band (i.e., flat bands) alongside Dirac bands in a reciprocal space, as revealed by TB band structure derived from hopping parameters of $t_{1}=t_{2}=t_{3}=-0.5 \mathrm{eV}$. In contrast, the Kagome-honeycomb lattice can be virtually derived from the Kagome lattice by introducing an additional bond between frustrated Kagome's triangles, associated with the additional hopping constant $t_{4}$. The TB band structure of the Kagome-honeycomb lattice (Fig. 4E) with hopping constants of $t_{1}=t_{2}=t_{3}=t_{4}=-0.5 \mathrm{eV}$ exhibits two flat bands below and above Dirac bands, which can be attributed to a pair of the intense peaks $\left(F B_{1}\right.$ and $\left.F B_{2}\right)$ in the electronic spectrum of the circumcoronenebased superlattice on $\mathrm{Cu}(111)$ (Fig. $4 \mathrm{~F}$ ).

The electronic Kagome-honeycomb lattice emerges as "antilattice" to the circumcoronene superlattice, whereby individual molecules act as a hexagonal repulsive barrier for 2DEG. The underlying physical picture is remarkably similar to the artificial electronic lattices (37-43) patterned by CO molecules on the $\mathrm{Cu}(111)$ surface via STM manipulation, wherein each CO molecule also acts as a repulsive scatter for 2DEG. Although STM manipulation endows an ultimate control over the lattice topology, this approach is unsuitable for practical applications due to a lack of scalability. Our findings surpass this limitation, paving a viable route toward a scalable bottom-up fabrication of the extended 2D electronic lattices, where NG with tailored topology is used as a "building block." We envisage that a rational design of NGs with different size and edge topologies can potentially serve as a versatile "tuning knob" for the efficient modulation of the hopping strength and electronic structure of artificially engineered electronic lattices.

We have demonstrated a conceptually new on-surface synthesis route toward the ultrahigh-yield fabrication of the hexagonalshaped circumcoronene molecule on $\mathrm{Cu}(111)$ via surface-assisted intramolecular cyclodehydrogenation of the rationally designed precursor molecule. A simultaneous assembly of large-area circumcoronene array results in a periodic confinement of the 2DEG into a unique chiral electronic Kagome-honeycomb lattice associated with the peculiar electrostatic potential landscape of the circumcoronene array on $\mathrm{Cu}(111)$. Our results open up a new avenue for high-yield fabrication of atomically precise elusive NGs with desired topologies toward large-scale fabrication of artificial 2D lattices with possible nontrivial electronic properties. The 2D Kagomehoneycomb lattices with a flat-band electronic structure can be particularly interesting in a wide scope of condensed matter physics, because of their prosperous potential in the realization of a variety of exotic manybody phenomena, including anomalous quantum Hall states (44-46), Wigner crystallization (47), and topological insulating transitions (48).

\section{MATERIALS AND METHODS}

\section{The qPlus STM/ncAFM measurements}

The STM and ncAFM experiments were performed under UHV conditions at $4.4 \mathrm{~K}$ using a commercial Omicron LT STM/AFM machine. We used the commercial qPlus sensor (49) with a resonant frequency of $f_{0}=28 \mathrm{kHz}$, a stiffness of $k=1800 \mathrm{Nm}^{-1}$, and a quality factor of 11,500 , which was operated in the frequencymodulation mode with an oscillation amplitude of $A=100 \mathrm{pm}$. All simultaneous STM/ncAFM images were collected in constant height mode. The tip apex was routinely decorated by a CO molecule by its controllable picking from the bare $\mathrm{Cu}(111)$ surface.

\section{Sample preparation}

$\mathrm{Cu}(111)$ single crystal (MaTeck $\mathrm{GmbH}$ ) was cleaned by multiple cycles of $\mathrm{Ar}^{+}$sputtering and annealing. The precursor molecules were deposited from the Knudsen cell (MBE-Komponenten $\mathrm{GmbH}$ ) at $350^{\circ} \mathrm{C}$ onto the clean $\mathrm{Cu}(111)$ surface under UHV conditions (base pressure $<3 \times 10^{-9} \mathrm{mbar}$ ). After the deposition of the precursor molecules, the sample was annealed at $140^{\circ} \mathrm{C}$ for 1 hour for the fabrication of circumcoronene arrays. Subsequently, the sample was transferred into the STM/AFM head held at a temperature of $4.5 \mathrm{~K}$ for ncAFM/STM imaging and characterization.

\section{DFT simulations}

DFT calculations were performed using the FHI-aims code (50) and Perdew-Burke-Ernzerhof generalized gradient approximation of the exchange-correlation potential $(51,52)$ to obtain the electronic properties of the circumcoronene molecule on the $\mathrm{Cu}(111)$ surface. The Tkatchenko-Scheffler treatment of the van der Waals (vdW) interactions was used during the calculations (53). We modeled the $\mathrm{Cu}(111)$ surface by a $3 \times 3$ supercell composed of three layers. After placing the molecule on the surface, we performed structural relaxations of all the atoms of the slab, except the last $\mathrm{Cu}$ layer. The optimization procedure was stopped when the total energy and the remaining atomic forces were found below $10^{-5} \mathrm{eV}$ and $10^{-2} \mathrm{eV} / \AA$, respectively. A Monkhorst-Pack grid of $3 \times 3 \times 1$ was used to integrate the Brillouin zone. Furthermore, the calculated total charge density and the Hartree potential were used to analyze the electronic interactions between the circumcoronene and the surface.

\section{TB calculations}

We carried out calculations of a TB model using the nearest-neighbor interaction between nodes of the Kagome-honeycomb lattice shown in Fig. 4E. All on-site energies have been set to zero and the nearest-neighbor hopping was set to $t=-0.5 \mathrm{eV}$ to mimic the experimental evidence. To obtain the band plot shown in Fig. 4E, TB Hamiltonian was diagonalized along a dense set of high-symmetry k-points.

\section{SPM simulations}

AFM image simulations were performed on the basis of the Probe Particle code $(28,29)$, which includes vdW and electrostatic interactions between the CO tip and the surface. Lennard-Jones potential was used for the treatment of Pauli repulsion and vdW interactions. The Hartree potential obtained from DFT calculations was used to calculate the electrostatic forces. All the simulations were done with an effective atomic radius $R_{\mathrm{c}}=1.661 \AA$ and a lateral stiffness of $k=0.25 \mathrm{~N} / \mathrm{m} . \mathrm{d} I / \mathrm{d} V$ maps were simulated using the PP-STM code (30) with a CO rigid tip (without tip-apex relaxation). We first calculated the eigenstates of the circumcoronene orbitals (with the FHI-aims code) that were subsequently used in the simulations. The $\mathrm{d} I / \mathrm{d} V$ maps were obtained at characteristic energy positions corresponding to frontier orbitals $(-1.6 \mathrm{~V},+0.9 \mathrm{~V}$, and $+1.8 \mathrm{~V})$. 
Note that the Lorentzian width parameter for broadening of the eigenstates was set to be $0.001 \mathrm{eV}$.

\section{SUPPLEMENTARY MATERIALS}

Supplementary material for this article is available at http://advances.sciencemag.org/cgi/ content/full/7/3/eabf0269/DC1

\section{REFERENCES AND NOTES}

1. S. Clair, D. G. de Oteyza, Controlling a chemical coupling reaction on a surface: Tools and strategies for on-surface synthesis. Chem. Rev. 119, 4717-4776 (2019).

2. L. Talirz, P. Ruffieux, R. Fasel, On-surface synthesis of atomically precise graphene nanoribbons. Adv. Mater. 28, 6222-6231 (2016).

3. M. Treier, C. A. Pignedoli, T. Laino, R. Rieger, K. Müllen, D. Passerone, R. Fasel, Surfaceassisted cyclodehydrogenation provides a synthetic route towards easily processable and chemically tailored nanographenes. Nat. Chem. 3, 61-67 (2011).

4. Q. Shen, H.-Y. Gao, H. Fuchs, Frontiers of on-surface synthesis: From principles to applications. Nano Today 13, 77-96 (2017).

5. J. Cai, P. Ruffieux, R. Jaafar, M. Bieri, T. Braun, S. Blankenburg, M. Muoth, A. P. Seitsonen, M. Saleh, X. Feng, K. Müllen, R. Fasel, Atomically precise bottom-up fabrication of graphene nanoribbons. Nature 466, 470-473 (2010).

6. O. Gröning, S. Wang, X. Yao, C. A. Pignedoli, G. Borin Barin, C. Daniels, A. Cupo, V. Meunier, X. Feng, A. Narita, K. Müllen, P. Ruffieux, R. Fasel, Engineering of robust topological quantum phases in graphene nanoribbons. Nature 560, 209-213 (2018).

7. P. Ruffieux, S. Wang, B. Yang, C. S. Sánchez, J. Liu, T. Dienel, L. Talirz, P. Shinde, C. A. Pignedoli, D. Passerone, T. Dumslaff, X. Feng, K. Müllen, R. Fasel, On-surface synthesis of graphene nanoribbons with zigzag edge topology. Nature 531, 489-492 (2016).

8. J. Su, M. Telychko, P. Hu, G. Macam, P. Mutombo, H. Zhang, Y. Bao, F. Cheng, Z.-Q. Huang, Z. Qiu, S. J. R. Tan, H. Lin, P. Jelínek, F.-C. Chuang, J. Wu, J. Lu, Atomically precise bottom-up synthesis of $\pi$-extended [5] triangulene. Sci. Adv. 5, eaav7717 (2019).

9. D. J. Rizzo, G. Veber, T. Cao, C. Bronner, T. Chen, F. Zhao, H. Rodriguez, S. G. Louie, M. F. Crommie, F. R. Fischer, Topological band engineering of graphene nanoribbons. Nature 560, 204-208 (2018).

10. S. Fuji, T. Enoki, Nanographene and graphene edges: Electronic structure and nanofabrication. Acc. Chem. Res. 46, 2202-2210 (2012).

11. S. Mishra, D. Beyer, K. Eimre, S. Kezilebieke, R. Berger, O. Gröning, C. A. Pignedoli, K. Müllen, P. Liljeroth, P. Ruffieux, X. Feng, R. Fasel, Topological frustration induces unconventional magnetism in a nanographene. Nat. Nanotechnol. 15, 22-28 (2020).

12. S. Zhao, J. Lavie, L. Rondin, L. Orcin-Chaix, C. Diederichs, P. Roussignol, Y. Chassagneux, C. Voisin, K. Müllen, A. Narita, S. Campidelli, J.-S. Lauret, Single photon emission from graphene quantum dots at room temperature. Nat. Commun. 9, 3470 (2018).

13. B. Cirera, A. Sánchez-Grande, B. de la Torre, J. Santos, S. Edalatmanesh, E. Rodríguez-Sánchez, K. Lauwaet, B. Mallada, R. Zbořil, R. Miranda, O. Gröning, P. Jelínek, N. Martín, D. Ecija, Tailoring topological order and $\pi$-conjugation to engineer quasi-metallic polymers. Nat. Nanotechnol. 15, 437-443 (2020),

14. C. Moreno, M. Vilas-Varela, B. Kretz, A. Garcia-Lekue, M. V. Costache, M. Paradinas, M. Panighel, G. Ceballos, S. O. Valenzuela, D. Peña, A. Mugarza, Bottom-up synthesis of multifunctional nanoporous graphene. Science 360, 199-203 (2018).

15. W. L. Wang, O. V. Yazyev, S. Meng, E. Kaxiras, Topological frustration in graphene nanoflakes: Magnetic order and spin logic devices. Phys. Rev. Lett. 102, 157201 (2009).

16. W. Han, R. K. Kawakami, M. Gmitra, J. Fabian, Graphene spintronics. Nat. Nanotechnol. 9 , 794-807 (2014).

17. F. Lombardi, A. Lodi, J. Ma, J. Liu, M. Slota, A. Narita, W. K. Myers, K. Müllen, X. Feng, L. Bogani, Quantum units from the topological engineering of molecular graphenoids. Science 366, 1107-1110 (2019).

18. M. Slota, A. Keerthi, W. K. Myers, E. Tretyakov, M. Baumgarten, A. Ardavan, H. Sadeghi, C. J. Lambert, A. Narita, K. Müllen, L. Bogani, Magnetic edge states and coherent manipulation of graphene nanoribbons. Nature 557, 691-695 (2018)

19. N. Koch, G. Heimel, J. Wu, E. Zojer, R. L. Johnson, J.-L. Brédas, K. Müllen, J. P. Rabe, Influence of molecular conformation on organic/metal interface energetics. Chem. Phys. Lett. 413, 390-395 (2005).

20. M. Treier, P. Ruffieux, R. Schillinger, T. Greber, K. Müllen, R. Fasel, Living on the edge: A nanographene molecule adsorbed across gold step edges. Surf. Sci. 602, L84-L88 (2008).

21. G. Li, Y. Han, Y. Zou, J. J. C. Lee, Y. Ni, J. Wu, Dearomatization approach toward a superbenzoquinone-based diradicaloid, tetraradicaloid and hexaradicaloid. Angew. Chem. Int. Ed. 58, 14319-14326 (2019).

22. L. Gross, F. Mohn, N. Moll, P. Liljeroth, G. Meyer, The chemical structure of a molecule resolved by atomic force microscopy. Science 325, 1110-1114 (2009).
23. L. Gross, Recent advances in submolecular resolution with scanning probe microscopy. Nat. Chem. 3, 273-278 (2011).

24. D. G. de Oteyza, P. Gorman, Y.-C. Chen, S. Wickenburg, A. Riss, D. J. Mowbray, G. Etkin, Z. Pedramrazi, H.-Z. Tsai, A. Rubio, M. F. Crommie, F. R. Fischer, Direct imaging of covalent bond structure in single-molecule chemical reactions. Science 340, 1434-1437 (2013).

25. A. Riss, A. P. Paz, S. Wickenburg, H.-Z. Tsai, D. G. de Oteyza, A. J. Bradley, M. M. Ugeda, P. Gorman, H. S. Jung, M. F. Crommie, A. Rubio, F. R. Fischer, Imaging single-molecule reaction intermediates stabilized by surface dissipation and entropy. Nat. Chem. $\mathbf{8}$, 678-683 (2016).

26. M. Di Giovannantonio, X. Yao, K. Eimre, J. I. Urgel, P. Ruffieux, C. A. Pignedoli, K. Müllen, R. Fasel, A. Narita, Large-cavity coronoids with different inner and outer edge structures. J. Am. Chem. Soc. 142, 12046-12050 (2020).

27. I. Pozo, Z. Majzik, N. Pavliček, M. Melle-Franco, E. Guitián, D. Peña, L. Gross, D. Pérez Revisiting kekulene: Synthesis and single-molecule imaging. J. Am. Chem. Soc. 141, 15488-15493 (2019).

28. P. Hapala, G. Kichin, C. Wagner, F. S. Tautz, R. Temirov, P. Jelínek, Mechanism of highresolution STM/AFM imaging with functionalized tips. Phys. Rev. B 90, 085421 (2014).

29. P. Hapala, R. Temirov, F. S. Tautz, P. Jelínek, Origin of high-resolution IETS-STM images of organic molecules with functionalized tips. Phys. Rev. Lett. 113, 226101 (2014).

30. O. Krejčí, P. Hapala, M. Ondráček, P. Jelínek, Principles and simulations of high-resolution STM imaging with a flexible tip apex. Phys. Rev. B 95, 045407 (2017).

31. L. Gross, N. Moll, F. Mohn, A. Curioni, G. Meyer, F. Hanke, M. Persson, High-resolution molecular orbital imaging using a $p$-wave STM tip. Phys. Rev. Lett. 107, 086101 (2011)

32. S. Wang, L. Z. Tan, W. Wang, S. G. Louie, N. Lin, Manipulation and characterization of a periodical graphene structures created in a two-dimensional electron gas. Phys. Rev. Lett. 113, 196803 (2014)

33. C.-H. Park, S. G. Louie, Making massless Dirac fermions from a patterned two-dimensional electron gas. Nano Lett. 9, 1793-1797 (2009).

34. I. Piquero-Zulaica, J. Lobo-Checa, A. Sadeghi, Z. M. Abd El-Fattah, C. Mitsui, T. Okamoto, R. Pawlak, T. Meier, A. Arnau, J. E. Ortega, J. Takeya, S. Goedecker, E. Meyer, S. Kawai, Precise engineering of quantum dot array coupling through their barrier widths. Nat. Commun. 8, 787 (2017).

35. D. Leykam, A. Andreanov, S. Flach, Artificial flat band systems: From lattice models to experiments. Adv. Phys. X 3, 1473052 (2018)

36. F. C. de Lima, G. J. Ferreira, R. H. Miwa, Topological flat band, Dirac fermions and quantum spin Hall phase in 2D Archimedean lattices. Phys. Chem. Chem. Phys. 21, 22344-22350 (2019).

37. K. K. Gomes, W. Mar, W. Ko, F. Guinea, H. C. Manoharan, Designer Dirac fermions and topological phases in molecular graphene. Nature 483, 306-310 (2012).

38. R. Drost, T. Ojanen, A. Harju, P. Liljeroth, Topological states in engineered atomic lattices. Nat. Phys. 13, 668-671 (2017).

39. M. R. Slot, T. S. Gardenier, P. H. Jacobse, G. C. P. van Miert, S. N. Kempkes, S. J. M. Zevenhuizen, C. Morais Smith, D. Vanmaekelbergh, I. Swart, Experimental realization and characterization of an electronic Lieb lattice. Nat. Phys. 13, 672-676 (2017).

40. S. N. Kempkes, M. R. Slot, S. E. Freeney, S. J. M. Zevenhuizen, D. Vanmaekelbergh, I. Swart, C. Morais Smith, Design and characterization of electrons in a fractal geometry. Nat. Phys. 15, 127-131 (2019)

41. L. C. Collins, T. G. Witte, R. Silverman, D. B. Green, K. K. Gomes, Imaging quasiperiodic electronic states in a synthetic Penrose tiling. Nat. Commun. 8, 15961 (2017).

42. S. E. Freeney, J. J. van den Broeke, A. J. J. van der Veen, I. Swart, I. C. Morais Smith Edge-dependent topology in Kekulé lattices. Phys. Rev. Lett. 124, 236404 (2020).

43. S. N. Kempkes, M. R. Slot, J. J. van den Broeke, P. Capiod, W. A. Benalcazar, D. Vanmaekelbergh, D. Bercioux, I. Swart, C. Morais Smith, Robust zero-energy modes in an electronic higher-order topological insulator. Nat. Mat. 18, 1292-1297 (2019).

44. H. Wakao, T. Yoshida, H. Araki, T. Mizoguchi, Y. Hatsugai, Higher-order topological phases in a spring-mass model on a breathing Kagome lattice. Phys. Rev. B 101, 094107 (2020).

45. K. Sun, Z. Gu, H. Katsura, S. Das Sarma, Nearly flatbands with non-trivial topology. Phys. Rev. Lett. 106, 236803 (2011).

46. G. Xu, B. Lian, S.-C. Zhang, Intrinsic quantum anomalous hall effect in the Kagome lattice $\mathrm{Cs}_{2} \mathrm{LiMn}_{3} \mathrm{~F}_{12}$. Phys. Rev. Lett. 115, 186802 (2015).

47. Y. Chen, S. Xu, Y. Xie, C. Zhong, C. Wu, S. B. Zhang, Ferromagnetism and Wigner crystallization in Kagome graphene and related structures. Phys. Rev. B 98, 035135 (2018).

48. H.-M. Guo, M. Franz, Topological insulator on the Kagome lattice. Phys. Rev. B 80, 113102 (2009).

49. F. J. Giessibl, The qplus sensor, a powerful core for the atomic force microscope. Rev. Sci. Inst. 90, 011101 (2019).

50. V. Blum, R. Gehrke, F. Hanke, P. Havu, V. Havu, X. Ren, K. Reuter, M. Scheffler, Ab initio molecular simulations with numeric atom-centered orbitals. Comp. Phys. Comm. 180, 2175-2196 (2009). 
51. A. D. Becke, Density-functional thermochemistry. III. The role of exact exchange J. Chem. Phys. 98, 5648-5652 (1993).

52. P. J. Stephens, F. J. Devlin, C. F. Chabalowski, M. J. Frisch, Ab initio calculation of vibrational absorption and circular dichroism spectra using density functional force fields. J. Phys. Chem. 98, 11623-11627 (1994).

53. A. Tkatchenko, M. Scheffler, Accurate molecular van der Waals interactions from groundstate electron density and free-atom reference data. Phys. Rev. Lett. 102, 073005 (2009).

54. A. Lerchen, S. Vásquez-Céspedes, F. Glorius, Cobalt(III)-catalyzed redox-neutral synthesis of unprotected indoles featuring an N-N bond cleavage. Angew. Chem. Int. Ed. 55, 3208-3211 (2016).

\section{Acknowledgments}

Funding: J.L. acknowledges support from MOE grants (MOE2017-T2-1-056 MOE2019-T2-2-044, R-143-000-A75-11, and R-143-000-B58-114). P.M. and P.J. acknowledge support from project no. 18-09914S of the Czech Science Foundation, Praemium Academie of the Czech Academy of Sciences, and Czech Nano Lab Research Infrastructure supported by MEYS CR (LM2018110). J.W. acknowledges financial support from NRF Investigatorship (NRF-NRFI05-2019-0005) and MOE Tier 2 grant (MOE2018-T2-2-094). M.T. acknowledges support from A*STAR under its AME YIRG grant (project no. A20E6c0098). Author contributions: J.L. supervised the project. M.T. performed experiments related to on-surface synthesis, STM/ncAFM measurements, and data analysis. G.L. and J.W. designed and synthesized the precursor molecules. P.M. and P.J. performed the DFT calculations. D.S.-P. and P.J. performed TB calculations. X.P., J.S., S.S., M.J.K., and M.E. contributed to the scientific discussion. The manuscript was written by M.T. and J.L., with contributions from all coauthors. Competing interests: The authors declare that they have no competing interests. Data and materials availability: All data needed to evaluate the conclusions in the paper are present in the paper and/or the Supplementary Materials. Additional data related to this paper may be requested from the authors.

Submitted 29 September 2020

Accepted 20 November 2020

Published 15 January 2021

10.1126/sciadv.abf0269

Citation: M. Telychko, G. Li, P. Mutombo, D. Soler-Polo, X. Peng, J. Su, S. Song, M. J. Koh, M. Edmonds, P. Jelínek, J. Wu, J. Lu, Ultrahigh-yield on-surface synthesis and assembly of circumcoronene into a chiral electronic Kagome-honeycomb lattice. Sci. Adv. 7, eabf0269 (2021). 